\title{
Exploration of Application and Practice of Numerical Control Technology in Machine Manufacturing
}

\author{
Chunfang Gao, Chunxia Shi
}

Baoji Vocational Technology College, Baoji Shaanxi, 721013, China

\begin{abstract}
Key words: Machine manufacturing, Numerical control technology, Application, Trend.
\end{abstract}
\begin{abstract}
With improvement of computer technology and digital technology level, the superiority of numerical control technology application in machine manufacturing gradually stands out. Numerical control technology optimizes equipment effect and quality, promotes market competitiveness of enterprises and drives leapfrog development of machine manufacturing industry. This paper briefly analyzes operation model of numerical control technology and discusses application process and development trend of numerical control technology in machine manufacturing so as to offer valuable reference for technical management personnel in machine manufacturing project.
\end{abstract}

\section{Introduction}

The development of modern machine manufacturing project presents a coercive trend. Traditional technological parameters and operation technology cannot adapt the demand of new products. Thus, relevant researchers need to establish complete technology upgrade planning according to actual conditions, and apply development framework of numerical control technology to promote actual quality of machine manufacturing. Equipment operating efficiency can improve, and products manufactured can conform to quality standards. In addition, numerical control technology can smoothly promote fusion of technology project and industrial development, enhance machine manufacturing structure level and boost market competitiveness of enterprises.

\section{Overview of numerical control technology}

\section{Development history of numerical control technology}

Development history of numerical control technology has presented high-speed development state in recent years. In about 1938, fast data operation and transmission project also showed a rapid development trend, which laid the operation foundation for computer numerical control technology. In 1952, the first set of numerical control machine tool was born in machinery industry.

\section{Connotation and operation principle of numerical control technology}

During studying numerical control technology, it is required to guarantee integrity of relevant operation parameter and structure, and start from technical level of numerical control technology in order to achieve entire technical parameter upgrade. Numerical control technology mainly utilizes computer technology and traditional machining process to enhance control of construction process. In technical operation process, operation mechanism of machinery project improves with the help of speed control, angle control and position control to make sure the operation framework of numerical control technology complies with actual project demand. In addition, the wide operation range of numerical control technology is based on its high efficiency and high precision advantages. Numerical control technology can effectively promote automation process and ensure important value of modern integration system.

Moreover, in order to achieve effective fusion of numerical control operation and computer technology, it is required to make sure smooth implementation of automatic control and machine manufacturing operation with the help of electric drive and precise measurement structure in the 
operation process of numerical control technology so as to achieve optimal operation of modern numerical control system. It is especially necessary to notice that during technology operation and promotion, management personnel should carry out different operation control of different models of machine tools so as to achieve optimization of entire control. In numerical control system, basic components include numerical control device, input device, output device, software operation device and speed control unit structure. Special electronic computer structure upgrade control mechanism is applied to ensure optimal operation of entire electrical elements, and real-time monitoring efficiency and operation precision improve. Meanwhile, efficient fusion of electronic computer structure and numerical control technology is guaranteed. The machine tool utilizes strong current control operation assist function to execute detection items of mechanism position and achieve signal and information transmission with the help of speed control unit and input/output channel.

\section{Equipment for numerical control technology operation}

During analyzing numerical control technology, numerical control technology equipment should be deconstructed to really achieve high-precision operation, except knowing the operation principle and basic parameter structure. Firstly, automatic control and intelligent digital servo technology. With the help of intelligent technology parameters, numerical control technology achieves internal power and electronic technology upgrade and avoids the defects of temperature drift and zero drift. Secondly, precision machinical technology. The technology should be detailed to precision machinery design and precision machinery processing, and ensure optimal operation of entire operation technology. In the composition conditions of numerical control machine tool, mechanical structure and transmission device are necessary components and have very crucial functions. Thirdly, precision detection and intelligent sensing technology. In the operation process of numerical control machine tool, entire operation structure upgrade can be achieved with the help of control measures to guarantee optima operation of component and sensor. Especially in the operation process of numerical control machine tool, the sensor can be used to accurately and quickly capture information so as to ensure effective operation of numerical control machine tool in different environment ${ }^{[1]}$.

\section{Application process of numerical control technology in machine manufacturing project}

\section{Operation framework of numerical control technology in industrial production}

With technological progress and development of society and economy, the application fields of numerical control technology become wider and wider. Its application in industry is prominent. Especially in modern industry, industrial development course can be effectively promoted only through improving integrity of entire operation parameters for modern industrial machine manufacturing project under the background of increasing market competition pressure and continuous upgrade of technical information structure. In the operation process of modern industrial project, operating environment of mechanical devices is quite inconsistent. Some devices need to operate in complex and adverse environment. Thus, challenges are proposed for operation validity and quality of devices. Operating personnel should enhance effective fusion of equipment operation structure and technology project in order to guarantee safety and standard of operation structure and working course. Automatic control structure and technical features can well complete the above requirements. Besides, in industrial production process, system program operation structure can effectively improve with the help of control unit based on basic components of computer. Furthermore, internal drive unit is used to establish effective transmission instruction according to information requirement so as to complete originally planned operation process and achieve real-time motoring. It is especially necessary to notice that in practice operation process, wrong operation signal or action instruction may encounter. The system will carry out information transfer through sensor device. After analyzing the feedback information, management personnel can intensively protect control unit structure and operation framework, and promote establishment of effective 
integral structure between entire servosystem and mechanical component of machine tool. Thus, the application of numerical control technology in industrial production can ensure large-scale improvement of production efficiency, achieve optimal personnel allocation, improve industrial level and enhance project safety and quality.

\section{Operation framework of numerical control technology in automobile industry}

In recent years, automobile industry in China has presented a high-speed development trend. People's demand for automobile is rising. Thus, new requirements are put forward for automobile operation technology. Not just should automobile appearance be guaranteed, but also overall performance should improve. Meanwhile, the speed, environmental protection structure and internal control performance of automobile should conform to standards. In automobile production process, new technology should be applied in component manufacturing to guarantee high-speed production of parts and components. The application of numerical control technology aims to promote production of components and accessories in automobile industry, and optimize automobile production quality and efficiency on the basis of improving automobile performance. In addition, the application of numerical control technology in automobile industry is to combine flexible management of production line and efficient operation technology, which achieves component operation technology upgrade and ensures fusion of processing technology and numerical control technology to some degree $^{[2]}$.

\section{Operation framework of numerical control technology in mechanical equipment production}

For each industry, mechanical equipment is the operation base. Operation framework of numerical control technology can comply with industrial development demand only when numerical control technology and mechanical equipment production framework are fused together. In the operation process of numerical control technology, it is first required to make processing preparation well, investigate basic parameters and operation conditions of machine tool equipment and establish effective analysis report in order to ensure complete working procedure. Then, professional personnel should carry out basic procedure testing of equipment to achieve effective trial processing, improve fusion of technology information and geometrical information, guarantee complete operation procedure and upgrade operation efficiency. After all preparations are done well, intensive summarization should be conducted for system operation framework. Then, formal processing can be implemented. After the completion of processing, intensive treatment and detection of system parameter and operation framework should be conducted to ensure optimal operation framework of equipment.

In mechanical equipment production, effective operation of computer control structure can improve the precision of automation project and efficiency upgrade, and guarantee more efficient parts machining process. Furthermore, the application of numerical control technology in equipment operation can improve mechanical equipment production and manufacturing efficiency, simplify original construction process and construction operation parameters, ensure great promotion of machine operation controllability and guarantee equipment quality to certain degree ${ }^{[3]}$.

\section{Operation framework of numerical control technology in coal mine mechanical equipment production}

Coal mine industry is an important industrial chain in China. To guarantee its operation efficiency and quality, it is required to make sure the whole industry advances stably on the basis of conforming to era development demand. Enhancing coal mining machinery with numerical control technology can guarantee smooth implementation of engineering project. There are any varieties of coal mining machines, and the development speed of different technical projects is fast in the development process. In traditional production process, processing technology cannot ensure weldment blanking timeliness of coal mine machinery, while modern numerical control technology can achieve the operation project that traditional operation structure cannot complete. Timely blanking for roller and 
blade structure of coal mining machine with keel plate procedure can enhance entire technical operation efficiency and operation structure. The application of numerical control technology in coal mine machinery processing project can maximally meet modern development demand, optimize operation quality reliability and speed, ensure maximum upgrade of operation advantage, really achieve integrity of operation structure of parts welding groove and guarantee integrity of cutting operation framework ${ }^{[4]}$. Besides, in project operation process, numerical control technology can maximally improve entire protection efficiency, guarantee automatic adjustment of joint-cutting compensation, and enhance overall control structure and management quality with the help of device features. Especially, for optimal control of workblank, integrity of overall parameter structure can improve, and work efficiency and quality of coal mine machinery production can be upgraded smoothly.

\section{Analysis of development trend of numerical control technology in machine manufacturing}

The application of numerical control technology is an inevitable trend of era development, not just because it has automation control advantage, but also because its technical features can ensure project quality while improving work efficiency. In the application process of numerical control technology, the combination of computer technology and digital operation framework can make sure its superiority is displayed visually. It can not merely improve integrity of platform operation framework process, but also guarantee convenient operation and management of project cost performance and really upgrade management structure, effectiveness and practical value of management parameter. In addition, the application of numerical control technology in machine manufacturing can bring changes from data and information level. As well, the application of numerical control technology in aerospace industry has been a mainstream operation technology in recent years, and can achieve integrity of parts processing project and production structure and make sure detailed parameters of parts comply with conventional market demand ${ }^{[5]}$.

Many traditional technologies cannot reach the advantages and characteristics of numerical control technology, so the importance of numerical control technology in machine manufacturing industry cannot be replaced by other technologies. The application of advanced numerical control technology can improve entire management mechanism and management parameters, ensure technical integrity, enhance cost performance, guarantee system operation process integrity, really achieve both-way upgrade of intelligence and automation and ensure synchronous improvement of quality and efficiency. Hence, it has extensive application prospect. Management personnel of numerical control technology should establish specific analysis mechanism for specific problems, optimize and upgrade technical operation parameters as well as make sure the structure can smoothly promote market competitiveness of enterprises. In addition, numerical control technology can guarantee high precision and high speed, realize high flexibility to certain degree, smoothly enhance project adaptation and make sure machining project development demand can be met in the operation process of numerical control technology. It is required to enhance integrity of automatic control structure and processing system in the process of optimizing numerical control technology. Structure parameters, actual size and weight of machine tool should be analyzed. Based on promoting system operation precision and system operation structure, equipment service life should effectively improve. Based on enhancing basic technical project, era characteristics of machinery industry should improve so as to really promote high-speed development of machinery industry.

\section{Conclusion}

In one word, machine manufacturing is a basic industry in China. Although it starts late, the core technology has been in development and progress. With numerical control technology, the operation efficiency of the industry and equipment quality can promote. So, numerical control technology deserves great promotion. In a bid to further improve development level of machine manufacturing 
industry, technical personnel should improve operation quality of technology, strengthen effectiveness of technical operation structure, establish complete development framework, facilitate integrity of technical operation process, start from actual operation perspective of industry, establish scientific operation process and drive sustainable development of machine manufacturing industry.

\section{Acknowledgments}

This paper is one of research achievements of 2016 education and scientific research topic of Shaanxi Society of Technical and Vocational Education - Study on Knowledge Module System Construction for Electronic Technical Skill Competition (No.: SZJY-1679)

\section{References}

[1] Liu Pai, On development of numerical control technology and its application in machine manufacturing. Mechanical and Electrical Information, 2013,15(03):101-102.

[2] Li Qingyong, Dou Meiling, Analysis of application of numerical control technology in machine manufacturing. Technology Innovation and Application, 2016,39(23):168-168.

[3] Sun Quanxi, Yu Hongge, Liu Zelin et al., Development and application prospect of numerical control technology in modern machine manufacturing. Inner Mongolia Science Technology \& Economy, 2015,40(24):73-73.

[4] Liu Congjun, On application of numerical control technology in machine manufacturing. Science and Technology, 2015,25(07):52-52.

[5] Gu Pingqing, Development trend and countermeasures of numerical control technology and equipment. 2014 nationwide summit forum for advanced manufacturing technology of mechanical equipment (Guangzhou) - collected papers of the 15th mechatronic engineering technology and application seminar in Guangdong and Hong Kong, 2014:421-423. 